\title{
L'enchaînement de la violence familiale et conjugale. Les grossesses hors mariage et ruptures du lien social au Burkina Faso
}

\section{Fatoumata Ouattara et Katerini Storeng}

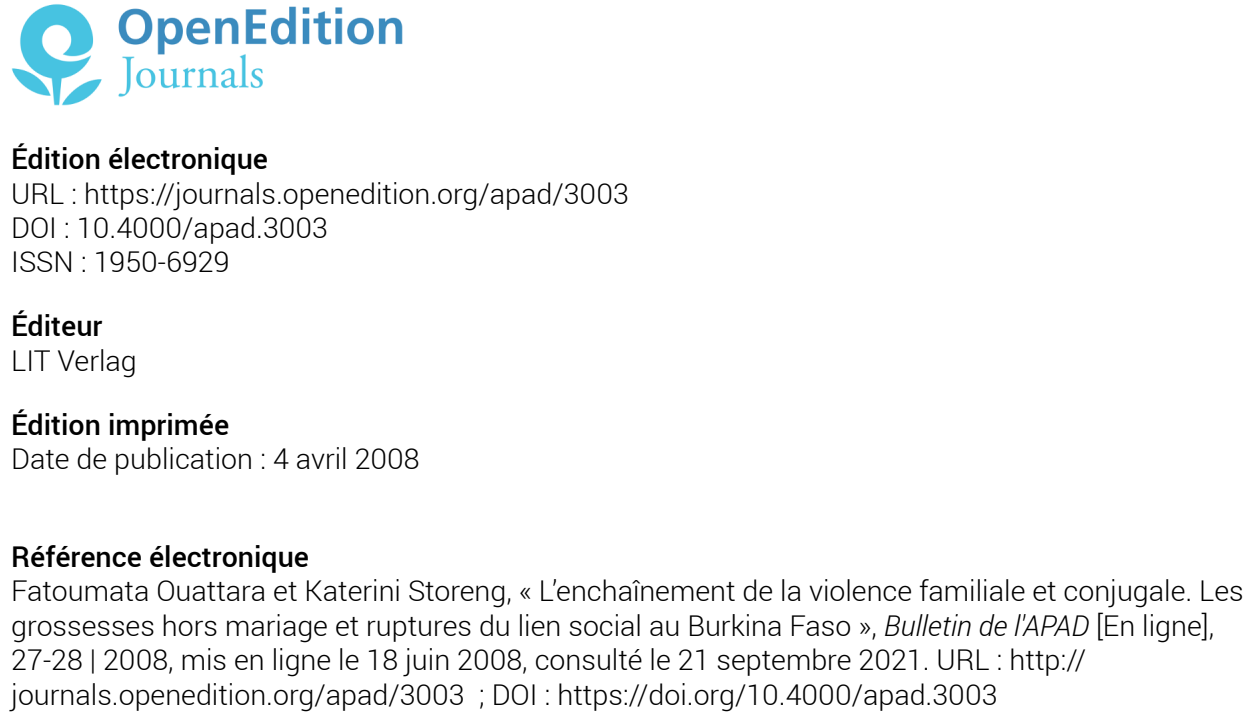

Édition électronique

URL : https://journals.openedition.org/apad/3003

DOI : 10.4000/apad.3003

ISSN : $1950-6929$

Éditeur

LIT Verlag

Édition imprimée

Date de publication : 4 avril 2008

Référence électronique

Fatoumata Ouattara et Katerini Storeng, « L'enchaînement de la violence familiale et conjugale. Les grossesses hors mariage et ruptures du lien social au Burkina Faso », Bulletin de I'APAD [En ligne], 27-28 | 2008, mis en ligne le 18 juin 2008, consulté le 21 septembre 2021. URL : http:// journals.openedition.org/apad/3003; DOI : https://doi.org/10.4000/apad.3003

Ce document a été généré automatiquement le 21 septembre 2021.

Bulletin de l'APAD 


\title{
L'enchaînement de la violence familiale et conjugale. Les grossesses hors mariage et ruptures du lien social au Burkina Faso
}

\author{
Fatoumata Ouattara et Katerini Storeng
}

\begin{abstract}
«(...) en définissant la structuration susceptible de participer à l'adaptation des individus à divers risques et à leur inscription sociale, on peut en déduire que les situations de vulnérabilité sont les circonstances - au sens de moments spécifiques et d'espaces déterminés - au cours desquelles cet exercice vital est plus particulièrement pénible, difficile ou périlleux. » (Delor \& Hubert, 2000 : 9)
\end{abstract}

1 Notre propos consiste ici à analyser les situations de violence qui affectent le lien de parenté et le lien conjugal qui jalonnent la trajectoire de construction d'une identité féminine. S'agissant du statut reconnu de la femme dans les sociétés africaines, d'abondantes descriptions ethnographiques ont montré que la légitimité sociale de la femme passait par le mariage et la fécondité. Or, les matériaux recueillis et mobilisés ici mettent plutôt en évidence des situations de violence qui s'apparentent à des contestations sociales de grossesse et/ou de projet de couple chez certaines femmes.

2 L'argument que nous voudrions développer ici pose que le processus de construction identitaire en mobilisant des stratégies individuelles d'affiliation à un réseau (le groupe d'alliance ou ceux qui en assument le rôle) entraîne un risque de désaffiliation par rapport à un autre réseau (le groupe de parenté). Mais de façon concomitante, la vulnérabilité relationnelle dans le groupe de parenté tend à fragiliser les relations avec les alliés qui deviennent tendues à leur tour. C'est alors que la violence peut apparaître. Dans les discours, tout acte de violence est présenté simultanément comme la conséquence d'une violence et la cause de celle qui va suivre. Comment comprendre ces enchaînements de violence? Quelles sont les justifications données à cette difficulté des rapports interpersonnels tant dans une parentèle, que dans le couple polygamique? Quels sont les répertoires normatifs ou idéels mobilisés pour légitimer ces actes de violence? Quelle interprétation sociologique donner à cette forme 
d'interaction? Comment produisent-elles du sens le processus d'individualisation qui travaille une société en crise? Comment le lien social s'entretient-il dans un environnement rendu particulièrement violent par la précarité économique? Ces formes de violence interviennent dans le cadre de l'application de sanctions consécutives à la transgression des normes sociales. Elles expriment la contestation de conduites sociales relatives à un statut singulier mis en jeu lors des relations (Demeulenaere 2003). La reproduction de la violence vient du fait que les différents statuts sont imbriqués (pour ne pas dire enchaînés) les uns dans les autres. Le processus de consolidation d'une position sociale au sein de la parentèle en dit long sur la qualité de l'insertion et la légitimité dans la relation conjugale. Le processus de la construction identitaire au sein de la famille n'est pas autonome de celui dans le couple. Les rapports violents (explicite ou implicite) participent au processus de légitimation des rapports conjugaux dans le couple, des rapports d'inégalité constitutifs de la parenté (aînés/ cadets), plus généralement des rapports de genre, et surtout des rapports de pouvoir et d'intégration sociale entre ceux qui disposent de ressources monétaires et ceux qui n'en disposent pas. En outre, l'émergence de la violence semble conditionnée par des situations de dépendance prenant diverses formes, et notamment celle liée à l'incapacité financière.

3 Les séquences d'une trajectoire féminine structurée à partir des expériences de grossesse et d'accouchement serviront de trame à notre analyse ${ }^{1}$. Nous convenons que l'usage du matériau biographique induit un risque de dilution de la question théorique aux dépens de la description "d'une expérience singulière " (Passeron $\left.{ }^{2} 2006: 2\right)$. La mobilisation de ces données biographiques s'accompagne ici d'une interprétation des "flux sociaux" qui participent à la structuration de la légitimation de la violence. Comment la trajectoire d'un acteur social s'inscrit-elle dans la «topographie et les calendriers institutionnels »? «Le temps du devenir social des individus et des groupes est, avant toute possibilité de choix tactique ou stratégique, déjà structuré par des normes, des définitions sociales, des représentations ou, plus généralement encore, des chances objectives, socialement conditionnées, de déroulement ou d'orientation biographique, "probabilités a posteriori », calculables au départ pour tout individu en fonction de ses appartenances...». (Ibid :10).

4 Comment s'élabore l'inscription des normes sociales dans les trajectoires d'une vie ? En définitive, nous userons d'une approche durkheimienne qui nous éclairera sur l'inscription "topographique " des normes. La carrière individuelle illustre superbement «une succession d'actions, réactives, défensives, tactiques, anticipatrices" choisies par l'acteur social pour faire face aux normes sociales contraignantes. En somme, elle correspond à un lieu de conjugaison de la "singularité du devenir d'un individu » et «la structure d'un temps social » (Ibid : 12).

La violence symbolique consécutive à l'anticipation de la fécondité sur le mariage

5 La trilogie "Femmes, mariage, maternité » nous est parfois présentée comme le parcours idéal-type de la réussite sociale féminine. De ce point de vue, le mariage et l'expérience de la maternité seraient les voies de reconnaissance sociale de la valeur féminine. À propos de la valorisation sociale de la femme africaine à travers sa capacité féconde, des anthropologues écrivaient ceci : «Dans les sociétés traditionnelles, ce sont les capacités reproductrices de la femme qui déterminent le statut auquel elle peut prétendre. (...) Son statut social évolue en fonction des étapes de sa vie génésique. La femme stérile, dans ce système, est dramatiquement "auréolée » d'indifférence ou, 
dans certains cas, accusée de sorcellerie. On dit parfois qu'elle «mange » la force vitale des enfants des autres femmes. Chez les Mossi, une femme sans enfant est une « femme vide ». C'est dire que la représentation de la femme est celle d'une bonne mère qui n'interrompt jamais le cycle de la reproduction. Elle féconde, elle accouche, elle allaite, etc. Il est même fréquent d'entendre dire qu'une femme « a soit un enfant au dos, soit un enfant dans le ventre » (Desjeux C. \& B., Bonnet D. 1983, ouvrage non paginé).

Convenons qu'au cours de nos différentes enquêtes, ces images «idéales» de la maternité ont été reproduites par les femmes, aussi bien en milieu urbain qu'en milieu rural et ce quel que soit leur groupe ethnique, leur âge, leur statut matrimonial et leur expérience génésique. La grossesse et l'accouchement sont toujours définis comme des étapes fondamentales et nécessaires dans la vie d'une femme: "toute femme doit connaître la douleur de l'accouchement ». C'est donc la valeur accordée à la fécondité qui est ici soulignée. Celle-ci est définie comme un besoin nécessaire à l'épanouissement d'une femme. En outre, l'accouchement permet à une femme d'acquérir de la reconnaissance, de la considération et du respect au sein de son entourage. Ce respect est souvent marqué par le fait que la femme est désignée par le nom de ses enfants : "la mère de... ». À l'inverse, la stérilité est perçue comme un malheur, une source de préoccupation, de tristesse et de dépression dans la vie d'une femme. Du fait de son infécondité, la femme peut être confrontée aux moqueries et aux railleries de l'entourage du couple. Plusieurs femmes ont cité en exemple, les affronts qu'une femme peut adresser à sa coépouse stérile quand elle cherche à commissionner un enfant. Leurs discours insistent sur la confrontation permanente de la femme inféconde à la "méchanceté » des autres femmes. On voit ici se profiler la menace de violence qui prend la forme du harcèlement verbal à l'encontre des femmes mariées dont la fécondité n'est pas attestée. Les récits de relations entre coépouses foisonnent de marques de stigmatisation à l'égard de celles qui n'ont pas d'enfant. Rumeurs et propos insultants sur leur infécondité confortent ces femmes dans la culpabilité.

7 En contraste avec ces représentations typiques et idylliques de la féminité, les récits des expériences de la grossesse et de l'accouchement ne sont pas exempts de situations de vulnérabilité. La violence sociale y trouve l'occasion de s'inscrire dans l'anticipation des statuts de la femme. Et parmi ceux-ci, l'expérience obstétricale est une circonstance privilégiée. Les relations interpersonnelles fondées sur la diversité des statuts (sœur, femme mariée, mère) mettent la femme dans une vulnérabilité relationnelle. Entendons par vulnérabilité relationnelle, le produit d'un processus de rupture de liens engendrant des rapports de désaffiliation (Valérie Cohen, «la vulnérabilité relationnelle »). La vulnérabilité n'existe donc que dans le contexte de relations sociales au cours desquelles l'acteur social (il s'agit ici de la femme) développe des stratégies de "prise de distance » par rapport aux attentes des autres, attentes qu'elle peut avoir intériorisé comme une obligation de rôle féminin! soit, inversement, elle peut tenter de "réduire la distance » - construite par les attentes - des autres qui la tient à l'écart d'attributs de rôles très valorisés localement ${ }^{3}$.

8 L'anticipation de la fécondité sur le mariage est un facteur d'exposition à la vulnérabilité relationnelle et par conséquent au risque de violence ${ }^{4}$. La survenue d'une grossesse chez une femme non mariée l'engage, en général, dans une série de rapports difficiles avec les membres de sa famille. La trajectoire génésique de cette jeune femme qui commence par inverser l'ordre des choses débute mal! Etre enceinte en dehors du mariage est un problème pour sa famille. En témoigne l'histoire de Marie. Avant de 
vivre à Ouagadougou, Marie vivait à Gaoua chez ses parents. Elle raconte qu'elle y avait un copain qu'elle aimait mais ses parents n'approuvaient pas leur relation. Ils justifiaient leur opposition par le fait que Marie était encore trop jeune pour se marier. Cependant en dépit de la désapprobation de ses parents, la jeune femme vécut une relation amoureuse avec son copain pendant deux ans (première forme de violence). Sa première grossesse arriva au cours de cette cohabitation et la rupture avec son copain intervint peu de temps après la survenue de cette première grossesse qu'elle qualifie d'« accident » dans la mesure où elle ne la souhaitait pas. À 20 ans, elle accoucha donc d'une fille (actuellement âgée de 6 ans et qui vit à Gaoua chez ses grands parents maternels).

9 La vulnérabilité de la jeune femme est marquée par le fait que contrairement aux espoirs de ses parents, elle se retrouve mère alors qu'elle n'est pas mariée. À l'instar d'autres sociétés sub-sahéliennes, le mariage des filles constitue un critère d'honorabilité pour leurs familles. Inversement, la honte peut advenir du fait qu'une fille « en âge de se marier " ne le soit pas. Cette honte est encore plus grande quand une grossesse survient avant le mariage. Car de façon paradoxale, si la fécondité est valorisée localement, la survenue d'une grossesse avant la demande en mariage est désapprouvée : « La priorité d'un agencement social sur un autre ne peut exister que si les gens intériorisent l'importance de l'ordre social en place et de son respect... » (Boutte 1998: 47). Deux années après cette rupture sentimentale et un premier accouchement, Marie fait la connaissance d'un homme qui passait un bref séjour à Gaoua. C'est l'homme chez qui nous l'avons rencontrée à Ouagadougou. Peu de temps après leur rencontre, elle décide de rejoindre son nouveau compagnon à Ouagadougou sans toutefois s'encombrer d'un accord parental. Le comble est qu'elle profite d'un voyage de sa mère partie à Bobo-Dioulasso pour des funérailles pour lui subtiliser des pagnes et des ustensiles de cuisine pour s'installer dans sa nouvelle vie de concubinage (deuxième forme de violence). Or c'est justement cette forme d'accouplement libre qui est réprouvée par les parents. Mais il y a plus, quand il n'y a pas de mariage, étant données les valeurs dominantes de l'idéologie locale, les familles ne peuvent pas s'allier ni se retrouver pour sanctionner positivement l'association des familles à travers l'union des deux conjoints.

10 Au-delà du mariage, de l'alliance et de l'honneur qui y est associé, qui va obliger les deux conjoints à suivre les normes ? En effet, ce n'est pas le Droit de la famille (ignoré de tous), mais les responsables des familles alliées qui peuvent contraindre les conjoints à respecter les conventions locales et à remplir leurs obligations de rôle. Hors de ce cadre normatif, l'individu « libre » n'est pas protégé par son groupe, il est soumis à tous les arbitraires et reste sans défense contre les abus possibles. Le fait, pour Marie, de prendre ses distances et d'imposer sans négociation son choix à sa parentèle, enclenche un processus de désengagement réciproque des membres de sa parentèle qui ne se sentent plus solidaires du destin de la jeune femme. En arrivant à Ouagadougou, Marie découvre que, contrairement à ce qu'il lui avait fait croire, son nouveau compagnon a déjà deux épouses (troisième forme de violence). La surprise est totale pour les deux coépouses qui n'ont pas été informées à l'avance de l'arrivée d'une «troisième » épouse (quatrième forme de violence). Alors, contrairement à l'usage, Marie n'est pas " accueillie » comme " une petite sœur » mais comme une rivale et une intrigante. Très vite, l'agressivité et les conflits s'installent dans les rapports quotidiens entre la nouvelle venue et ses coépouses. Le mari qui les a toutes trompées, la protège contre les autres femmes. Procédure classique. Les modalités d'insertion de la nouvelle femme 
justifient l'ampleur des réactions violentes de ses coépouses et contribuent à la rendre vulnérable. Contrairement aux normes locales concernant la régulation des relations entre coépouses, l'arrivée de Marie n'a pas été annoncée à ses coépouses. Il n'est donc pas étonnant que la coépouse qui aurait dû être perçue comme une "petite sœur » soit vite devenue la rivale, celle que l'on doit écarter à tout prix et si possible faire partir. Des deux coépouses, la deuxième était la plus réticente à l'arrivée d'une troisième. En effet, dans l'idéologie polygamique, la deuxième femme est considérée comme la favorite et l'arrivée d'une troisième épouse ne peut que susciter des stratégies de protection de privilèges acquis ou considérés comme tels. Ses multiples conflits avec l'homme et avec la troisième lui coûtèrent sa place (sixième forme de violence). Elle perdit la bataille. Nonobstant le départ d'une des deux premières épouses, les relations ne s'apaisèrent jamais entre Marie et la première femme qui menaçait de l'attaquer par des pouvoirs magiques. (septième forme de violence).

Quelques temps après ces menaces d'attaques en sorcellerie (explicites ou implicites), Marie fut prise de troubles physiologiques. C'était en 2003 et la seule parente de Marie qui réside dans le quartier se souvient : «Elle est tombée gravement malade. Quand elle est hors de la cour, elle est en bonne santé, quand elle vient, elle rentre dans la cour, elle ne peut pas dormir, et elle ne peut pas dormir, elle va crier comme ça jusqu'au matin. (...) Elle avait mal partout et quand elle rentrait à la maison, on aurait dit qu'elle allait mourir même, souvent, elle allait dormir dans les six mètres (rues au sein des quartiers lotis), sinon quand elle était dans la cour-là, c'était grave.» Le mari croit également à une attaque sorcière de la part de sa première femme. Il encouragera alors la jeune femme à quitter le domicile pendant une bonne partie de la journée. Marie le suivait jusqu'à son lieu de travail qui consiste à faire payer ses services comme un intermédiaire obligé entre les propriétaires de véhicules et les agents d'un service de transports réservé à la régularisation des documents d'achats de véhicules. La parente de Marie ne manqua pas une occasion de lui signaler sa désapprobation quand elle constata que la complicité du couple était telle que Marie suivait chaque jour son homme à son lieu de travail (tentative de rappel à l'ordre normatif). L'empiètement sur les territoires masculins est prioritairement décrié par les femmes et non comme on aurait pu s'y attendre par des hommes. La légitimation des rapports de genre prend toute sa valeur avec leur intériorisation par les femmes. Dominées, ce sont elles qui paradoxalement, se placent en position de veiller à la reproduction des conditions de leur subordination.

12 La menace et la crainte d'attaque en sorcellerie et la suspicion permanente que cette situation engendre, saturent les vécus quotidiens des femmes dans les couples polygames (Fainzang et Journet 1988 : 116-120). L'entourage partage ces conceptions. La parente de Marie raconte que c'est par crainte que Marie ne meure ou ne devienne folle qu'elle lui a suggéré de retourner vivre au village. Mais elle a refusé. Dans sa belle-famille, les choses ne vont guère mieux. Selon la « sœur » de Marie là-bas « elle ne s'entend avec personne dans sa belle-famille, personne ne l'aime dans sa belle-famille, (...) elle n'est soutenue par personne ». Lors de notre première rencontre, à la question de savoir si tout se passait bien dans la cour avec les personnes qui y vivaient, elle n'hésitait pas à répondre par la négative. Selon elle, les membres de sa belle-famille ne l'aimaient pas parce qu'elle n'était pas de la même ethnie qu'eux (elle est Dagara, eux sont Mossi) et qu'ils lui ont même suggéré de retourner vivre dans son village. Les tensions avec les membres de la belle-famille ont toujours existé, mais elles se sont exacerbées après le décès de sa belle-mère. En outre, une sœur de son mari récemment 
répudiée par son époux et venue s'installer chez eux et ne cachait pas son antipathie vis-à-vis d'elle (huitième forme de violence). En réalité lors de notre première visite dans la concession, cette mauvaise qualité des relations entre Marie d'un côté, sa coépouse et sa belle-sœur de l'autre était palpable. L'ambiance suspicieuse à l'égard de notre présence fut telle que la jeune femme décida contre toute attente que l'entretien ait lieu en français, une langue que ne comprenaient ni la coépouse ni la belle-sœur. Une exclusion linguistique qui, on peut l'imaginer, ne faisait qu'ajouter à la tension des rapports conflictuels entre les occupantes de l'espace domestique. Notons ici que Marie ne se privait pas de démontrer toute la fierté qu'elle retirait de notre visite à "son " domicile et la supériorité aussi que manifestait sa capacité de s'exprimer dans une langue valorisée localement. Les tensions entre les épouses et leurs belles-sœurs sont tout aussi banales que celles qui caractérisent les relations entre coépouses. Alors, la promiscuité dans l'espace de vie n'arrange pas les choses. Coépouses, enfants, maris, belles-sœurs, cousins partagent quotidiennement le même espace de vie domestique.

La mésentente entre coépouses est une structure relationnelle classique ${ }^{5}$. Cependant, la nature de la violence des rapports entre coépouses tient à l'histoire singulière de chaque couple polygamique. Les coépouses n'ont pas tardé à comprendre la vulnérabilité relationnelle de Marie, dont l'arrivée n'avait été légitimée par aucun rite de mariage. Or, celui-ci mobilise les familles, les clans, les générations, et scelle ainsi un pacte d'alliance qui joue, on l'a vu, comme une garantie collective qui protège la femme. Tout se passe comme si le mariage maintenait l'appartenance de la femme à son groupe de parenté tout en assurant son intégration au sein du groupe de ses beaux-parents. Mais Marie ne s'intéresse pas au mariage, elle transgresse toutes les conventions et ce faisant, elle déchante à partir de ses actes de violence initiaux une série d'actes de violence en retour (ici nous en avons souligné huit). En somme, aucun membre de la parenté de la jeune femme n'est connu dans la cellule conjugale. En dépit d'une absence de demande en mariage, elle se sépare des siens sans recevoir l'accord et le soutien de ses parents. Enfin, elle est d'autant plus dangereuse que son arrivée précipite la répudiation d'une des coépouses.

La violence de la désaffiliation du réseau de parenté

14 La violence familiale, structurelle, se concentre sur les acteurs dont la position est la plus vulnérable. L'inégalité des relations entre aînés et cadets qui structure la dynamique des réseaux de parenté s'entretient à l'aune des contraintes et des obligations sociales. Que celles-ci ne soient pas respectées et une réaction violente viendra du réseau de parenté mis à l'épreuve de la solidarité. L'infériorité statutaire, la faiblesse, la pauvreté suscitent ordinairement de la violence des supérieurs, des puissants et des riches (Janin 2003). Marie appartient à une fratrie composée de trois filles et d'un garçon. Ce dernier vit à Ouagadougou où il est agent de la fonction publique. Mais quand Marie arriva dans la capitale, contrairement aux règles du savoir-vivre, elle ne rendit pas visite à ses "parents». Elle ne se comporta pas en «bonne petite sœur». Les "parents» de la jeune femme lui reprochèrent le choix d'une vie égoïste. «Elle ne venait même pas ici, elle ne voulait même pas que quelqu'un sache qu'elle était à Ouagadougou ici, elle se cachait ». En mesure de rétorsion, cette femme qui se considérait comme sa "sœur» décida de ne pas lui rendre visite. Bien qu'elles résidassent dans le même quartier, ce n'est qu'après plusieurs visites de Marie que cette sœur se rendra à son tour chez Marie, avant de décider de mettre un terme à ses visites en raison des multiples conflits auxquels elle avait assisté entre Marie et son mari. Quant à son frère, Marie avait passé trois années à Ouagadougou sans s'être 
rendue chez lui. La première visite avait été recommandée par la «sœur » qui l'avait d'ailleurs accompagnée. Quand, pour une raison de maladie, sa mère séjourna chez son frère à Ouagadougou, ce fut la deuxième fois que Marie se rendait au domicile de son frère (celui-ci hébergeait la mère). Le frère profita de cette visite de Marie pour lui reprocher ses mauvais choix de vie et lui faire entendre qu'elle se faisait exploiter par les hommes qui lui faisaient des enfants. Irritée par ces paroles, Marie aurait alors «mal parlé » à son frère qui a alors juré de ne plus se considérer comme un frère pour elle.

Dans la société dagara, proférer de telles paroles à l'égard d'un membre de sa fratrie ou d'un descendant direct est un acte grave qui exige une réparation rituelle au village. Consécutivement à ce serment, le frère ne pouvait plus aider sa sœur en quoi que ce soit sous peine de mort pour parjure. Contrairement à ce que les membres de sa famille lui reprochaient, Marie nous confiait qu'elle rendait souvent visite à ses "parents " à Ouagadougou mais qu'en retour, ils ne venaient jamais la voir chez elle. Pour elle, la raison principale en étaient que : «Selon eux, mon mari n'est pas venu leur demander l'autorisation afin que je puisse rester chez lui. Je suis venue chez mon mari sans leur avis ». Mais en ne "recherchant» pas les siens en ville, Marie avait aussi enfreint une règle de bienséance élémentaire du lien social communautaire. La culpabilité de l'individu est imputée à son comportement individualiste. On se trouve ici dans la logique communautaire, telle que avancée par Alain Marie (2003) qui définit l'individu " comme perpétuellement en dette vis-à-vis du groupe, avec obligation réciproque d'être socialement altruiste, et, en complémentarité, parce qu'elle le soumet au refoulement de sa dimension la plus individuelle - son individualisme-, qui l'expose à la violence répressive. » (Marie $2003: 14$ ).

Mais en réalité, ces deux tendances ne sont pas aussi clairement distinctes dans les situations de la vie quotidienne. Les mêmes acteurs sociaux qui revendiquent la "valeur des traditions africaines" sont bien souvent les mêmes à mobiliser le changement, la modernité pour rendre légitime un style de vie et inversement. D'un point de vue normatif, la victime de la violence sociale est aussi un agresseur. La malédiction proférée par le frère rappelle insidieusement la préséance de la communauté sur la liberté individuelle. La trajectoire de Marie se situe au cœur de l'antagonisme entre des normes sociales de conduite ou de comportement définies comme immuables - "on est né trouver ça» - et des stratégies d'autonomie individuelles inscrites dans la trajectoire personnelle. Il est moins question de vérité objective ici que de vérité requise (Janin, 2003: 50). Comme on le verra plus loin, l'extrême vulnérabilité économique de la jeune femme dans une situation d'urgence sanitaire ne parviendra pas à faire "parjurer " son frère. On peut d'ores et déjà en déduire que ce n'est pas parce qu'un réseau de parentèle est disponible qu'il est forcément mobilisable ${ }^{6}$. La capacité de mobilisation de la solidarité dont la parentèle est porteuse dépendra étroitement de la qualité du lien entretenu avec ses membres plus précisément de l'évaluation par les membres du réseau de la conformité avec laquelle on s'acquitte de ses obligations de sociabilité. Car le «système de protection sociale » de la solidarité familiale n'est pas « automatique ». Les logiques sociales qui participent à la reconnaissance statutaire dans les liens de parenté se fondent sur une réciprocité où priment "les solidarités calculées et les dettes à répétition » (Vuarin 2000). Ainsi, pour que le principe de solidarité soit à l'œuvre, encore faut-il que son processus soit activité! Les droits acquis au titre de l'affiliation familiale ne 
déclenchent pas automatiquement le bénéfice d'une aide ou d'un soutien en retour qui devra être gagné par des efforts de sociabilité.

La solidarité se mérite par la conformité aux normes du jeu social : Le demandeur de soutien doit faire un choix stratégique et se conformer aux règles de préséance. S'il ne le fait pas, ainsi que ses parents le reprochent à Marie, alors la solidarité ne jouera pas et le demandeur d'aide va se trouver en situation de vulnérabilité. Être en situation de vulnérabilité, c'est se trouver dans l'incapacité de mobiliser les ressources adéquates (sociales, relationnelles, monétaires, etc.) pour faire face à une situation d'exposition à la souffrance ou à la violence, qui a pour conséquence un autre risque : celui d'en subir des conséquences personnelles très graves. Si vulnérabilité et culpabilité sont intrinsèquement liées dans la mise en pratique des violences ordinaires, le cadre des relations familiales semble propice à la culpabilisation des faibles. Nous conviendrons avec Pierre Janin de la faible capacité pour l'individu à transformer ses liens familiaux par une prise de distanciation engagée dans un processus d'individualisation (Janin 2003 : 34-35). À cet égard, la vulnérabilité relationnelle des conjoints en union libre est la conséquence directe de l'absence d'accord et d'implication de leurs familles respectives, leur choix et leur décision. Ainsi de façon concomitante, la vulnérabilité consécutive à une désaffiliation par rapport au groupe de parenté s'accompagnera d'un affaiblissement d'une non reconnaissance du lien conjugal par les beaux-parents : non mariée, le partenaire de l'homme demeure une usurpatrice qui n'existe pas socialement aux yeux des parents de cet homme. La vulnérabilité de la femme vivant ainsi en union libre se traduit par une angoisse permanente de se retrouver seule, à la rue. La précarité de leur projet de vie se traduit par une triple exposition de la femme au risque de violence conjugale, au risque de ne pas disposer des ressources nécessaires, ni de pouvoir les mobiliser (solidarité familiale) pour faire face à cette situation et enfin au risque d'en subir des conséquences graves pour sa vie. C'est ce qui arriva à Marie.

De la violence structurelle nichée dans la grossesse

18 Les situations de grossesse se conjuguent souvent à des situations de violence. Bien qu'il soit intrinsèquement douloureux pour la femme avant tout, l'échec d'une grossesse peut susciter la suspicion de sa belle-famille et la colère du compagnon. Ainsi, Marie a eu un fils avec son mari. Il avait trois ans au moment de l'enquête. Nous avons fait la connaissance de Marie 4 mois après la perte d'une grossesse qu'elle portait. Elle se plaignait de douleurs physiques en dépit des médicaments qui lui avaient été prescrits à l'hôpital. Elle racontait que pendant la grossesse, elle avait ressenti des douleurs après avoir transporté des charges d'eau de la fontaine du quartier. Puis un écoulement de sang s'en était suivi. Conduite dans un premier temps à la maternité du quartier où elle a passé plusieurs heures, elle fut ensuite envoyée à la maternité du centre hospitalier pour des soins d'urgence. La jeune femme fut sauvée de justesse. Son séjour hospitalier est marqué par les signes de l'exclusion. Ainsi, pendant son hospitalisation, des voisines avaient payé les ordonnances et sa parente avait payé les frais d'hospitalisation et le transport du taxi pour la conduire à l'hôpital central. Quant à la coépouse, elle n'apporta aucun soutien moral ni matériel à Marie. Elle ne prit aucune nouvelle de la malade et ne prit même pas la peine de lui envoyer un plat cuisiné à l'hôpital. Mais avant cela, pendant sa grossesse, ses rapports conjugaux étaient d'une grande violence, tant psychique que physique. Dans les souvenirs de la jeune femme, sa grossesse a été marquée aussi bien par le manque de nourriture que par d'incessants conflits entre elle et son mari. Elle confie avoir été battue à maintes reprises. En plus de cette violence physique, elle était victime de violence verbale. Il 
niait sa responsabilité dans la grossesse et clamait haut et fort que Marie avait " cherché » cette grossesse. Les actes de violence ne s'estompèrent pas avec l'arrêt de la grossesse. L'accusation changea simplement de prétexte. L'homme accusait Marie d'avoir interrompu volontairement sa grossesse. Il l'avait alors insultée, traitée de sorcière en la rendant coupable d'avoir provoqué la fausse-couche en ingurgitant des médicaments. Après cet épisode obstétrical douloureux, la jeune femme affirmait ne plus avoir envie de faire un autre enfant. Pourtant, les symptômes de souffrance physiologique que Marie évoquait pendant l'entretien faisaient immanquablement penser à un probable état de grossesse. Nos soupçons s'avérèrent justifiés quelques mois plus tard.

19 À l'instar du VIH/Sida (Castro et Farmer 2006), les femmes les plus vulnérables socialement et économiquement sont également celles qui sont le plus exposées à la non programmation des grossesses et à l'expérience des complications obstétricales. Bien qu'elles connaissent les risques de complications, elles disposent d'une autonomie financière limitée pour pouvoir accéder à temps à des soins de qualité. Ici comme ailleurs, la pauvreté marquée par la précarité au niveau de l'emploi, le manque de moyens, l'inégalité d'accès aux ressources matérielles ainsi que le manque du pouvoir de décision se conjuguent au féminin (Kinda 2000). Seule l'urgence mobilise les ressources financières: "quand ça chauffe, on trouve ». De manière générale, l'inégalité, quelles que soient ses formes et les rapports qui l'entérinent, peut produire de la violence ${ }^{7}$ (Héritier 1996 : 31). Cependant, on doit constater que dans le domaine de la santé, les analyses ont rarement pris en compte simultanément l'insertion individuelle dans le cycle d'accès aux ressources matérielles et dans le cycle de la reproduction (Devreux 2001). Or la fragilité physique se double d'une culpabilité fragilisante compromettant la solidarité conjugale. On a observé que la précarité de la relation sociale (l'inexistence d'un lien de mariage) aggravait la fragilisation des femmes en situations de complications obstétricales. Etre une jeune fille non mariée ou une femme vivant en concubinage constitue en soi une vulnérabilité au risque qui se cristallise avec la survenue de la grossesse et/ou d'une complication obstétricale (fausse-couche, mort-né, grossesse extra-utérine).

Après la fausse-couche, les relations entre Marie et son « mari » ne se sont pas apaisées. Elles se sont au contraire dégradées en s'exacerbant sur une accusation d'adultère. L'homme reprocha à sa femme d'avoir un amant. La parente de Marie rapporta qu'il l'avait surprise chez l'amant et l'avait même battue. «Il l'avait blessée, le lendemain, elle ne pouvait même pas se lever, tout son corps faisait mal, c'est le deuxième jour qu'elle a pu marcher... ». Ensuite, il a convoqué l'amant au commissariat de police, mais les policiers ont déconsidéré la plainte, car selon eux seul un acte de mariage civil entre Marie et son prétendu mari pouvait justifier d'une accusation d'adultère. L'affaire fut donc classée. L'amant en question était un marabout et Marie justifiait ses nombreuses visites chez lui par le fait qu'il était un client régulier de la cola qu'elle vendait et qu'il voulait aussi l'aider à trouver du travail. Notons que la parente de Marie désapprouvait la conduite de sa sœur et la traitait de femme aux "cuisses légères » qui ne savait pas contrôler ses désirs sexuels. « ... j’ai eu à lui dire que si c'est sur la route que ton mari te rencontrait et te frappait comme ça, il te voyait avec un homme et te frappait, moi je n'allais pas lui donner raison, mais comme il est parti te prendre, te surprendre dans une cour-là, je dis même que devant ton mari, moi je dis, je ne suis pas d'accord pour ton comportement (...). Sincèrement, ce qu'elle a vécu là-bas, ce n'était pas bon, ce n'était pas bon.» Elle alla jusqu'à qualifier la conduite sexuelle de Marie de 
«pathologique ». Elle nous apprit qu'à Gaoua, Marie avait "emménagé chez un guérisseur" de qui elle avait eu un enfant qui est décédé peu de temps après l'accouchement. Ensuite, elle avait encore déménagé pour aller chez un autre guérisseur de qui elle avait aussi eu un enfant (c'est la première fille de Marie âgée d'environ 6 ans et vivant à Gaoua).

21 Comme partout, l'adultère est pratiqué alors qu'il est réprouvé par toutes les normes sociales. La règle sociale en la matière consiste donc à être discret et à ne pas se donner à voir en public, au vu et au su de tout le monde, afin de préserver son honneur et celui d'autrui. Alors la pratique par Marie de rapports adultérins indiscrets, publics, est intolérable pour l'environnement social. La condamnation à subir des violences physiques et verbales est la réponse sociale donnée non point à la transgression de l'interdit de l'adultère, mais bien plus à la transgression de la norme de discrétion (Ouattara 1999, Kintz 1987). Quand Marie a accouché de sa dernière grossesse, son «mari » était en prison pour une accusation de détournement d'argent. Il y passa une dizaine de jours. En rentrant de son séjour pénitencier, il a renié la paternité du nouveau-né et pour "marquer le coup ", il a renoncé à donner un nom au bébé. Par conséquent, deux mois après l'accouchement, l'enfant ne portait toujours pas de nom ! La coépouse de Marie avait, elle, aussi à sa façon, condamné l'acte d'adultère qui avait produit un enfant. Ainsi, elle avait refusé de s'occuper du placenta ramené au domicile en arguant qu'elle n'avait pas à s'occuper d'un enfant qui n'était pas de son mari. «Ils ont amené le placenta à la maison, (...) la coépouse a dit que ça ne la regardait pas. (...) c'était posé là, jusqu'à la nuit (...) on a appelé des vieilles, elles sont venues enterrer ça. La coépouse a dit qu'elle ne toucherait pas au placenta, que ce n'est pas l'enfant de son mari... ». Avoir un enfant sans identité est en soi une sanction sociale très grave. Le refus de donner son identité à un être placé de fait sous sa responsabilité est une grande violence sociale et symbolique. Cette décision singulière récuse la logique normative qui établit qu'un homme soit le père de l'enfant de sa femme. Le processus de reconnaissance du lien filial qui légitime du même coup le lien conjugal est rompu. À ce égard, l'acte d'individuation de l'homme de Marie peut être interprété en même temps comme un acte de violence ordinaire et symbolique à l'égard de normes sociales établies.

La violence ordinaire à la croisée de la vulnérabilité économique

22 Les situations quotidiennes de la vie de couple de Marie sont chargées de violence ${ }^{8}$. Marie se fait régulièrement battre pour des motifs variés. Par exemple, les petites économies qu'elle cache dans sa chambre disparaissent régulièrement. Quand elle en parle à son «mari », celui-ci se sent accusé, il la bat non pas pour nier sa culpabilité, mais bien pour lui prouver qu'une femme ne doit pas accuser son mari de voleur. Accuser ouvertement le chef de famille en dépit de sa culpabilité porte préjudice à son honneur (Le Potvin 2005). Une autre fois, il arracha la porte de sa maisonnette pour l'exhorter à quitter la concession. Marie ira passer deux jours chez un cousin militaire, c'est celui-ci qui contraindra le compagnon à remettre la porte. Comme pour marquer son pouvoir d'homme et d'époux, il décide de confisquer la pièce d'identité de Marie pour réduire toute capacité de voyage. Marie sait que sans pièce d'identité, il lui sera difficile d'effectuer un quelconque voyage au cours duquel elle sera exposée au contrôle policier qui commence toujours par la demande des pièces d'identité. Refaire une autre carte d'identité exige une dépense. Or, la jeune femme a déjà des difficultés financières pour s'alimenter. Pendant notre entretien, elle semblait affamée et elle demandera à ce 
qu'on lui donne quelque chose pour diminuer sa faim. En dépit de la violence subie, Marie semble condamnée à être aliénée.

Les souffrances et les violences qu'elle subit ne sont pas dénoncées. La violence conjugale s'énonce en d'autres contextes. Marie est titulaire d'un diplôme obtenu à Gaoua dans un centre de formation ménager où elle a appris à faire la couture, la broderie, le tissage, la teinture. Un des projets du frère de Marie consistait à investir dans un télécentre que Marie devait gérer à Gaoua. Mais ce projet fut compromis par les projets de vie de Marie. «Il disait qu'il voulait ouvrir un télécentre à Dano là-bas pour elle, elle allait gérer, comme elle a au moins le certificat (études primaires), elle sait lire et puis écrire, bon tout ça là, c'est dans l'eau. » L'échec de ce projet nourrit sans doute l'âpreté des rapports entre Marie et son unique frère.

24 À Ouagadougou, Marie arrive à survivre grâce à de petits emplois précaires. Pendant quelques mois, elle faisait le porte à porte dans le quartier pour laver le linge dans les concessions. Puis, comme ce travail était épuisant physiquement, elle a entrepris de faire de la coiffure. Mais très vite, cette activité s'arrêta car au cours d'une bagarre avec son mari, elle eut un doigt blessé. Elle demanda à sa « sœur » de l'argent pour pouvoir vendre au détail des noix de cola. Il faut dire que l'entraide économique dans le réseau de parenté se limite à cette seule "parente", institutrice qui habite dans le même quartier que Marie. Même son hospitalisation pendant la fausse-couche n'engagera pas son frère à lui venir en aide. « ... elle a juré contre son grand frère, son grand frère n'a pas le droit de sortir (dépenser) 5F pour elle, il y a une affaire qu'il faut aller régler au village, tant que c'est pas résolu, si son grand frère s'amuse dans ça, il va mourir, donc il est obligé de la laisser comme ça, c'est moi seule qui suis en train de lutter avec elle...». L'intervention des ancêtres comme garants des rapports familiaux est ici revendiquée pour justifier la décision de ne pas accorder la solidarité à une "parente ». La connaissance d'un état d'urgence n'entraîne pas forcément la mobilisation de la solidarité familiale. La solidarité déclarée n'est pas automatique. Si solidarité il y a, elle est «calculée " et ses normes sociales posent l'individu dans une "obligation réciproque d'être socialement altruiste" (Marie 2003 : 14). Celui qui ne s'embarrasse pas de générosité prend le risque de ne pas recevoir d'entraide en situation de vulnérabilité. En définitive, se vérifie ici le constat fait par Robert Vuarin à Bamako (Vuarin 2000) selon lequel, et contrairement à ce que tout le monde dit, l'entraide sociale n'est pas orientée vers les plus démunis socialement et matériellement.

Un état de maladie aggravé, la dégradation d'un état de santé en situation de grossesse ne conduisent pas à une décision de recours aux soins. Une femme n'en vaut pas une autre dans le réseau de parenté ou dans un couple polygamique. Pour payer les soins consécutifs à la perte de sa grossesse, Marie a mobilisé ses économies (8000 FCFA). En revanche, les conseils médicaux recommandant le repos après sa sortie d'hôpital n'eurent pas d'échos. Deux semaines après la sortie de l'hôpital, elle reprit ses activités ménagères dont la plus laborieuse consistait à porter l'eau depuis la fontaine située à plusieurs centaines de mètres de son domicile. Elle ne peut ni compter sur une aide financière quelconque pour s'acheter de l'eau ni sur un soutien de sa coépouse pour la décharger d'une telle activité contraignante physiquement. Quant à son mari, sa situation financière est également précaire. Lors de leur première rencontre, il travaillait pour le compte d'une entreprise de travaux publics. À Ouagadougou, Marie le découvre intermédiaire dans les couloirs d'un service de transport où il fait payer ses services pour la constitution des dossiers de régularisation des véhicules importés. 
Mais les affaires ne marchent pas bien. Peu de temps après l'arrivée de Marie à Ouagadougou, il avait vendu une des concessions familiales pour pallier aux difficultés financières.

En dépit des difficultés quotidiennes et de la solitude, le retour « au village » ne semble pas représenter une alternative possible pour la jeune femme. Sans doute, la crainte de la réaction des parents restés au village n'encourage pas sa volonté de retour. N'oublions pas que Marie avait quitté Gaoua sans l'accord des siens. Comment entreprendre le chemin du retour après avoir défié ouvertement tous les siens ? Pour couronner son infortune, Marie ne ramènerait que son expérience d'un séjour en ville marqué par les actes de violence subis, la solitude, la précarité économique et aussi un " enfant qui n'a même pas de nom ». Pour réparer cette absence d'identité, une fois au village, le nom d'un « des petits frères au village " serait attribué au nouveau-né afin de permettre l'établissement d'un acte de naissance. Retour donc à la case départ! Craint et redouté, le village d'origine deviendrait ainsi le recours dès lors que disparaitraient les possibilités d'insertion dans un univers urbain devenu périlleux et laissant peu de place à l'insertion individuelle dans des réseaux d'entraide. De façon paradoxale, en même temps que l'univers urbain autorise l'individualisme en rendant possible la prise de distance à l'égard des normes, il permet aussi - à tout le moins pour les individus les plus vulnérables - des situations de solitude sociale extrême. L'intériorisation des normes et la justification consubstantielle des écarts de conduite légitiment le maintien des liens avec la famille et l'éventuel retour au « village » en situation de faillite sociale. «Dans son foyer sincèrement, vraiment, ce n'est même pas une vie, c'est une vraie souffrance (...) ». Cette proclamation de l'unique "parente» citadine de Marie insiste sur la spécificité de l'adversité que la jeune femme devait affronter dans une solitude révoltante. Une telle confession renvoie au caractère globalisant de la violence. Tout se passe comme si, la vulnérabilité induite par les relations avec les "parents » et la vulnérabilité due aux difficiles relations de couple, fonctionnaient dans un système de circularité.

Outre sa dimension sociale, ce qu'il semble important de souligner ici c'est la dimension justificatrice de la violence. Les conduites violentes requièrent des formes de légitimité de la part de leurs acteurs. Mais pour qu'elle soient légitimées, encore faut-il que les logiques sociales qui les sous-tendent soient régies par une intériorisation des normes sociales et une volonté de prise de distance à l'égard de ces normes : "Le schème de l'inconscient apporte une solution originale à la question de la mise en ordre parce qu'il permet de concevoir la contrainte sous la forme d'une puissance indissociablement extérieure et intérieure à la personne, comme une extériorité intériorisée : une force qui, si elle devait s'imposer de l'extérieur se manifesterait comme violence, vient habiter les personnes, les contraindre de l'intérieur, déterminer leurs conduites en épousant les contours de leur volonté. Elle tend à estomper la différence entre la violence physique et les autres formes de contraintes et, à la limite, à traiter sur un pied d'égalité toutes les déterminations, qu'elles soient ou non justifiables. » (Boltanski et Thévenot $1991: 419)$. En pratique, les formes de violences peuvent s'enchaîner et/ou alterner à travers des rapports sociaux mettant en cause le lien social familial ou conjugal. 


\section{BIBLIOGRAPHIE}

Boltanski Luc \& Thévenot Luc, 1991, De la justification. Les économies de la grandeur, Paris, Gallimard Boutte Joseph, 1998 « la violence ordinaire dans les villes subsahariennes », Cahiers de l’UCAC, n³ , p. $39-60$

Castro Arachu \& Farmer Paul, 2006, « Anthropologie de la violence : la culpabilisation des victimes », Notre Librairie, « penser la violence » (numéro spécial), $n^{\circ} 148$ (juillet-septembre 2002), p. 102-108

Cohen Valérie, «La vulnérabilité relationnelle. Essai de cadrage et de définition », Adresse : http ://revel.unice.fr/anthropo/document.html ?id=74; date de consultation : 05/10/06

Delor François \& Hubert Michel, 2003, « Un ré-examen du concept de « vulnérabilité » pour la recherche et la prévention du VIH/Sida », Observatoire du sida et des sexualités, p. 1-33 (publié dans Social Science \& Medicine, 50 (2000) , p. 1557-1570).

Demeulenaere Pierre, 2003, Les normes sociales. Entre accords et désaccords, Paris, PUF.

Desjeux C. \& B., Bonnet D., 1983, Africaines, paris, L'Harmattan.

Devreux Anne-Marie, 2001, «Les rapports sociaux de sexe : un cadre d'analyse pour des questions de santé ? », in Aïach P., Cèbe D., Cresson G., Philippe Cl. (eds), Femmes et hommes dans le champ de la santé. Approches sociologiques, Éditions de l'École Nationale de la Santé Publique, p. 97-116

Dubet François « Introduction à la problématique de la violence ». Conférence Symposium de Bruxelles, Source :www.chez.com. Date de consultation : 05/10/06. Adresse : http :// www.chez.com/b105/lectures/dubet/violence.htm.

Fainzang S., Journet O., 1989, La femme de mon mari. Anthropologie du mariage polygamique en Afrique et en France, Paris, L'Harmattan

Filippi V., Ronsmans C., Goufodji S., Lardi M., Sahel A., Saizonou J. \& De Brouwere V., 2005, «Maternal wards or emergency obstetric rooms? Incidence of near-miss events in African hospitals. » Acta Obstet Gynecol Scand, 84 (1), p. 11-16

Héritier Françoise (eds), 1996 De la violence, Paris, Éditions Odile Jacob

Janin Pierre et Marie Alain, 2003, « Violences ordinaires, violences enracinées, violences matricielles », Politique Africaine, $n^{\circ}$ 91, p. 5-12

Janin Pierre, 2003, « Vivre ensemble ou la douleur d'être « en grande famille » », Politique Africaine, $n^{\circ} 91$, p. $33-50$

Kinda Fatoumata, 2000, « Informations statistiques sur les femmes : la pauvreté au féminin au Burkina Faso », Colloque «Statistique, Développement et Droits de l'Homme », Montreux 4 $-8 / 09 / 2000$

Kintz Danièle, 1987, « De l'art peul de l'adultère », Bulletin de l'AFA, 29-30, p. 119-143

Le Potvin Sébastien, 2005, Les fils textuels des violences familiales au Mali, Master, Université de Provence, Aix-Marseille. 
Mantel, G.D., Buchmann, E., Rees, H., \& Pattinson, R.C. (1998). Severe acute maternal morbidity : a pilot study of a definition for a near-miss. British Journal of Obstetrics and Gynaecology, 105(9), 985-990.

Marie Alain (éd.), 1997, L’Afrique des individus, Paris, Karthala.

Marie Alain, 2003, « La violence faite à l'individu (La communauté au révélateur de la sorcellerie) », Politique Africaine, $\mathrm{N}^{\circ} 91$, p. 13-32

Ouattara Fatoumata, 1999, Savoir-vivre et honte chez les Senufo Nanerge (Burkina Faso), Thèse de doctorat (nouveau régime), EHESS-Marseille.

Passeron Jean-Claude, « Biographies, flux, trajectoires. Questions de l'extérieur. », Avant-propos, Enquête, $\mathrm{n}^{\circ} 5$ Biographie et cycle de vie. Adresse : http ://enquete.revues.org/document77.html, date de consultation : 05/10/06.

Reynaud Jean-Daniel, 1997, Les règles du jeu. L'action collective et la régulation sociale, Paris, Armand Colin/Masson.

Traoré Sarata, 2005, Mariage et célibat à Ouagadougou, Paris, L'Harmattan.

Vuarin R., 2000, Un système africain de protection sociale au temps de la mondialisation ou «venez m'aider à tuer mon lion... ", Paris, L'Harmattan.

\section{NOTES}

1. La trajectoire obstétricale décrite dans cet article a été reconstituée dans le cadre d'une étude sur les conséquences sociales des complications obstétricales (grossesses, fausses-couches, avortements et accouchements) effectuée dans le cadre du programme Initiative for Maternal Mortality Programm Assessment (IMMPACT) coordonné par l'université d'Aberdeen (Ecosse). L'étude sur les conséquences obstétricales (Outcomes After Pregnancy) fut coordonnée par la London School of Hygiène \& Tropical Medicine et le centre Muraz de Bobo-Dioulasso au Burkina.L'étude avait combiné une composante épidémiologique et une composante anthropologique à laquelle nous avons participé. Pour les besoins de l'enquête anthropologique, des entretiens approfondis ont été conduits avec des femmes retenues pour l'approche épidémiologique. Ce répertoire sélectif comprenait des femmes considérées comme des « échappées belles » ou Near-Miss (Mantel, G.D., Buchmann, E., Rees, H., \& Pattinson, R.C. (1998). Comparativement aux décès maternels, les cas de Near-Miss sont plus fréquents dans les hôpitaux africains. Par exemple, au Bénin, une étude a démontré que la survenue des complications obstétricales était 15 fois supérieure à celle des décès maternels (Filippi et al. 2005). L'étude réalisée au Burkina Faso a concerné des femmes ayant échappé de justesse à un décès durant la grossesse, l'accouchement ou le post-partum avec un enfant vivant ou mort-né ; des femmes qui avaient échappé à un décès de justesse après un avortement et des femmes qui avaient vécu un accouchement normal. Au moins deux entretiens ont été menés après la sortie d'hospitalisation avec les femmes retenues pour l'étude. Enfin des entretiens ont souvent eu lieu avec certains membres de leur entourage (conjoints ou " parents »).

2. http://enquete.revues.org/document77.html; page 2.

3. Si sa stratégie, quelle qu'elle soit, s'oppose aux attentes réciproques des autres, alors l'interaction va générer une tension, puis la succession d'interactions tendues va générer un conflit, plus ou moins ouvert, qui peut déboucher sur des actes de violence. 
Quoi qu'il en soit, le conflit et la violence ne conduisent pas nécessairement à la rupture de lien! Pour qu'il y ait rupture, il faut que l'un des protagonistes du conflit ne croit plus à la possibilité d'améliorer sa position, son statut, son rôle dans l'échange social avec l'autre, aussi conflictuel que soit cet échange. La rupture du lien, et de l'échange social qui va avec, se produit quand il devient plus intéressant pour l'un des protagonistes de sortir de la relation que d'y rester.

4. Certes, toutes les femmes désirent se marier. Mais une fois mariées, l'anticipation (qui est une attente forte en forme de pression) par le conjoint, par la belle-famille, par l'environnement social en général de la fécondité à venir, rend la femme nécessairement vulnérable. Cette vulnérabilité s'exprime par l'exposition permanente de la femme à des violences verbales ou comportementales comme le harcèlement psychologique, le mépris, le dénigrement, la stigmatisation, etc

5. Calquées sur les scènes de vie quotidiennes, les rivalités et méchancetés entre coépouses alimentent les récits romanesques africains (Le Potvin 2005).

6. Cohen, « la vulnérabilité relationnelle. Essai de cadrage et de définition », http ://revel.unice.fr/anthropolo/document.html ?id=74

7. Voir également Coomasraswany R., 1995, le rapport de l'ONU sur les violences contre les femmes, Genève.

8. Pour des récits variés à propos des rapports tendus et violents au sein des couples, voir Sarata Traoré (2005).

\section{RÉSUMÉS}

Cet article a pour objectif l'analyse de diverses formes de violence consécutives aux ruptures biographiques associées à l'expérience obstétricale dans une trajectoire de construction identitaire féminine. Diverses situations de violence s'enchaînent alors tant dans la désaffiliation du lien parental que dans les stratégies de construction d'un projet de couple. La violence apparaît alors dans la précarité relationnelle qui affecte les liens familial et conjugal devenus vulnérables. Si les champs sociaux ne sont pas distincts les uns des autres, les processus de reconnaissance sociale qui s'y rapportent confrontent l'acteur social à un risque de violence quand il se trouve en situation d'écart relatif aux normes sociales. L'enquête a montré que les femmes enceintes non mariées expérimentaient des rapports sociaux marqués par une violence extrême.

Title : The sequence of family and marital violence. Extra marital pregnancies and breaking-off of social link in Burkina Faso. The aim of this article is to analyse various forms of violence resulting from a breaking-off in the biographies related to obstetrical experience in the process of female identity building. Various situations of violence occur as well in the disaffiliation of the parental linkage as in the strategies to build a family. Violence manifests itself in the relational sphere that affects familial and conjugal relations that become vulnerable. If the social fields are not distinct from each other, the processes of social recognition which are related to them confront the social actor to a risk of violence when he is in a situation of limited distance to the social standards. This research has shown that especially non-married pregnant women experience social relations that are marked by extreme violence. 